\title{
Electric Field of the Ocean Induced by Diffusion
}

\author{
Pavel Tishchenko \\ Laboratory of Hydrochemistry, V.Il'ichev Pacific Oceanological Institute, Vladivostok, Russia \\ Email: tpavel@poi.dvo.ru
}

Received 28 December 2014; accepted 18 January 2015; published 21 January 2015

Copyright (C) 2015 by author and Scientific Research Publishing Inc.

This work is licensed under the Creative Commons Attribution International License (CC BY). http://creativecommons.org/licenses/by/4.0/

c) (i) Open Access

\begin{abstract}
The equations for gradient of electric field in seawater induced by gradients of salinity, temperature and pressure were developed by means of non-equilibrium thermodynamics. Extrathermodynamic assumptions and accepted chemical model of seawater permit to carry out numerical calculations of electric field caused by diffusion, thermodiffusion and barodiffusion for realistic hydrophysical structure of the ocean. It is shown that contribution of barodiffusion into electric field of the ocean is almost constant (about $-3 \times 10^{-7} \mathrm{~V} / \mathrm{M}$ ). This magnitude can be ignored in many cases because it is too small. However natural salinity and temperature gradients significantly impact into electric field of the ocean.
\end{abstract}

\section{Keywords}

Electric Field, Seawater, Diffusion, Thermodiffusion, Barodiffusion, Pitzer Method

\section{Introduction}

Natural electromagnetic fields in the ocean have two types of the sources: external (ionospheric and magnetospheric current systems) and internal one [1]. Internal source of electromagnetic field is the dynamo interaction of moving seawater with the Earth's magnetic field [2]. From this point of view, seawater is considered as conducting continuum only and physical theory is used for suggested experiments and for interpretation of experimental data [3] [4]. However seawater is electrochemical system (multicomponent electrolytes solution) which is not uniform regarding to temperature and concentrations (salinity). Also seawater is subjected hydrostatic pressure along depth. Thermodynamics of electrolyte solutions experimentally and theoretically well establishes existence electric fields induced by gradients of concentrations, temperature and pressure (for example, [5]). Nevertheless, experimental and theoretical considerations of the electrochemistry are never used for study of the electromagnetic fields in the ocean, excepting the case of preparation of electrodes, which are considered as "in- 
ert”. This paper attempts to partly eliminate gap between physics and electrochemistry in the study of electromagnetic field of the ocean. Some results have been published elsewhere [6] [7].

\section{Thermodynamic Background}

Three important fluxes occur in a multicomponent electrolyte solutions: those of matter, heat, and electricity. These three fluxes are described by well-known laws of Fick, Fourier, and Ohm as being proportional to appropriate thermodynamic force. The more general case, where interactions between these processes occur, leads to a set of simultaneous equations which are formulated by non-equilibrium thermodynamics [8].

Let us to consider seawater as $n$-components fully dissociated electrolyte solutions where each ion is assuming as component. When such electrolyte is subjected by thermodynamic forces then $n+1$ fluxes should be induced which defined by equations:

$$
\boldsymbol{J}_{i}=\sum_{j=0}^{n} l_{i j} \cdot \boldsymbol{X}_{j}
$$

where $\boldsymbol{J}_{i}$ is the flux of $i$ species; $\boldsymbol{X}_{j}$ is $j$ generalized thermodynamic force; $l_{i j}$ is the phenomenological coefficient which may be considered as generalized conductivity; index " 0 " corresponds to solvent (water). In further it will be used Hittorff's reference frame for fluxes which means that

$$
\boldsymbol{J}_{0}=0
$$

when system is in mechanical equilibrium. In the Equation (1) $n+1$ thermodynamic forces are connected by generalized form of Gibbs-Duhem equation:

$$
\sum_{j=0}^{n} m_{j} \boldsymbol{X}_{j}=0
$$

Here $m_{j}$ is molality of species $i$. Taking into account of Equations (2) and (3) the thermodynamic force and flux regarding to water can be excluded, then $n$ independent fluxes may be expressed via $n$ independent forces as follows

$$
\boldsymbol{J}_{i}=\sum_{j=1}^{n} L_{i j} \cdot \boldsymbol{X}_{j}
$$

Kirkwood et al. [9] demonstrated that when fluxes defined by the Hittorff's reference frame (Equation (4)) then Onsager's reciprocal relationships between phenomenological coefficients, $L_{i j}$ are fulfillment

$$
L_{i j}=L_{j i}
$$

Existence of temperature, concentration, and gravitational field defines thermodynamic forces and Equation (4) can be rewritten as follows [8]:

$$
\boldsymbol{J}_{i}=-\sum_{j=1}^{n} L_{i j} \cdot\left[M_{j} \boldsymbol{g}+z_{j} F \nabla \varphi+\bar{V}_{j} \nabla P+\eta_{i}^{*} \nabla T+\left(\nabla \mu_{j}\right)_{T P}\right]
$$

Here $M_{j}$ is molar mass of $j$ species; $\boldsymbol{g}$ is the downward directed acceleration due to gravitational field; $z_{j}$ is the charge number of ion $j ; F$ is Fraday number; $\varphi$ is electrical potential; $\bar{V}_{j}$ is partial molal volume of the $j$ ion; $\eta_{j}^{*}$ is the entropy of transfer [10]; $T$ is temperature in Kelvin scale; $\mu_{j}$ is chemical potential of $j$ ion; upward direction is accepted as positive. At non-steady states, the electroneutral rule for electrolyte solutions is following [10]:

$$
\boldsymbol{i}=\sum_{i=1}^{n} z_{i} \boldsymbol{J}_{i}=0
$$

where $\boldsymbol{i}$ is electrical current. From mechanical equilibrium condition, the relationship is following:

$$
\nabla P+\rho \boldsymbol{g}=0
$$

Here $\rho$ is density of the solution. From the Equations (6), (7) and taking into account (8) the equation for gradient of electrical field is following 


$$
\nabla \varphi=-\frac{1}{F} \frac{\sum_{i=1}^{n} z_{i} \sum_{j=1}^{n} L_{i j}}{\sum_{i=1}^{n} z_{i} \sum_{j=1}^{n} L_{i j} z_{j}}\left[\left(M_{j}-\bar{V}_{j} \rho\right) \boldsymbol{g}+\eta_{j}^{*} \nabla T+\left(\nabla \mu_{j}\right)_{T P}\right]
$$

Due to reciprocal Onsager's relationships (Equation (5)), the equation for transference number of species $i$ in Hittorff's reference frame, $\tau_{i}$, may be derived [11] as follows

$$
\frac{\tau_{j}}{z_{j}}=\frac{\sum_{j=1}^{n} L_{i j}}{\sum_{i=1}^{n} z_{i} \sum_{j=1}^{n} L_{i j} z_{j}}
$$

Taking into account that

$$
a_{i}=m_{i} \gamma_{i}
$$

the equation for gradient of the chemical potential is following:

$$
\left(\nabla \mu_{j}\right)_{T P}=R T\left(\frac{1}{m_{j}} \nabla m_{j}+\sum_{k=1}^{n} \frac{\partial \ln \left(\gamma_{j}\right)_{P T}}{\partial m_{k}} \nabla m_{k}\right)
$$

Here $a_{j}, \gamma_{j}$ are activity and activity coefficient of the $i$ ion, respectively; $R$ is gas universal constant. The final fundamental equation for gradient of electrical field in the electrolyte subjected under temperature, concentration and gravitational fields becomes from Equations (9)-(12)

$$
\nabla \varphi=-\frac{1}{F} \sum_{j=1}^{n} \frac{\tau_{j}}{z_{j}}\left[\left(M_{j}-\bar{V}_{j} \rho\right) \boldsymbol{g}+\eta_{j}^{*} \nabla T+R T\left(\frac{1}{m_{j}} \nabla m_{j}+\sum_{k}^{n} \frac{\partial \ln \left(\gamma_{j}\right)_{T P}}{\partial m_{k}} \nabla m_{k}\right)\right]
$$

\section{Numerical Calculations of Electric Fields Induced in Seawater by Temperature, Concentration and Gravitational Fields}

There are two problems of rigorous calculation of the Equation (13). Main problem is that thermodynamic properties of the individual ions cannot be strictly determined from thermodynamic point of view. Second problem is that there are a few experiments, which may to be used for numerical estimations of the Equation (13). Nevertheless, I try to carry out of numerical calculations of the Equation (13) using extrathermodynamic assumptions. These calculations have been approximated by empirical algorithms which provide a valuable tool for non-specialists in thermodynamics working in the other research of the electromagnetic fields of the ocean.

The main feature of the seawater is that molality of major constituents of seawater exhibit an almost constant ratios to one another throughout the oceans. Therefore it is can be written as follows:

$$
m_{i}=k_{i} S
$$

Here, $k_{i}$ is proportional coefficient for $i$ species. This feature permits to introduce salinity, $S$, into derivatives of chemical potential that simplifies the Equation (13)

$$
\nabla \varphi=-\frac{1}{F} \sum_{j=1}^{n} \frac{\tau_{j}}{z_{j}}\left[\left(M_{j}-\bar{V}_{j} \rho\right) \boldsymbol{g}+\eta_{j}^{*} \nabla T+R T\left(\frac{1}{S}+\frac{\partial \ln \left(\gamma_{j}\right)_{T P}}{\partial S}\right) \nabla S\right]
$$

Let us to introduce following notations:

$$
\begin{gathered}
\phi_{b d}=-\frac{g}{F} \sum_{j=1}^{n} \frac{\tau_{j}}{z_{j}}\left(M_{j}-\bar{V}_{j} \rho\right) \\
\phi_{t d}=-\frac{1}{F} \sum_{j=1}^{n} \frac{\tau_{j}}{z_{j}} \eta_{j}^{*}
\end{gathered}
$$




$$
\phi_{d}=-\frac{R T}{S F} \sum_{j=1}^{n} \frac{\tau_{j}}{z_{j}}\left(1+\frac{\partial \ln \left(\gamma_{j}\right)_{T P}}{\partial \ln S}\right)
$$

Here $g$ is the module of the $\boldsymbol{g} ; \phi_{b d}$ is the barodiffusion potential with dimension [V/M]; $\phi_{t d}$ is the thermodiffusion potential with dimension [V/grad]; $\phi_{d}$ is the diffusion potential with dimension [V/psu] where notation of psu (dimensionless "practical salinity unit") expresses salinity as a mass fraction in per mill.

\subsection{Calculation of the Diffusion Potential in Seawater}

For calculation diffusion potential, the composition of major constituents of seawater was taken from [12] and presented in Table 1. Transference numbers for given composition of seawater (Table 1) have been calculated from partial electrical conductances of major ions [13] and obtained values are listed in Table 1.

The derivatives of the activity coefficients of ions from salinity in the Equation (18) have been calculated by means of the Pitzer method (for example, [14] [15]). The Pitzer method starts with a virial expansion of the excess Gibbs energy, $G^{\text {ex }}$, of the solution. The expressions for activity coefficients are obtained by appropriate derivatives of excess Gibbs energy with respect to weight of the solution, $n_{W}$, and molality of the component $i$, respectively. Equations (19), (20) give the expressions for the activity coefficients of cations $M$, anions $X$ species, respectively.

$$
\begin{aligned}
\ln \gamma_{M}= & \frac{1}{R T} \frac{\partial G^{\mathrm{ex}}}{\partial m_{M}}=z_{M}^{2} F+\Sigma m_{a}\left(2 B_{M a}+Z C_{M a}\right)+\Sigma m_{c}\left(2 \Phi_{M c}+\Sigma m_{a} \psi_{M c a}\right)+1 / 2 \Sigma m_{a} m_{a^{\prime}} \psi_{M a a^{\prime}}+z_{M} \Sigma \Sigma m_{c} m_{a} C_{c a} \\
& +z_{M}\left\{\sum_{c} m_{c} \lambda_{c c} / z_{c}-\sum_{a} m_{a} \lambda_{a a} /\left|z_{a}\right|+(3 / 2) \sum_{c} \sum_{a} m_{c} m_{a}\left(\mu_{c c a} / z_{c}-\mu_{c a a} /\left|z_{a}\right|\right)\right\} . \\
\ln \gamma_{X}= & \frac{1}{R T} \frac{\partial G^{\mathrm{ex}}}{\partial m_{X}}=z_{X}^{2} F+\Sigma m_{c}\left(2 B_{X c}+Z C_{X c}\right)+\sum m_{a}\left(2 \Phi_{X a}+\Sigma m_{c} \psi_{X a c}\right)+1 / 2 \Sigma m_{c} m_{c} \psi_{X c c^{\prime}}+\left|z_{X}\right| \Sigma \Sigma m_{c} m_{a} C_{c a} \\
& +z_{X}\left\{\sum_{c} m_{c} \lambda_{c c} / z_{c}-\sum_{a} m_{a} \lambda_{a a} /\left|z_{a}\right|+(3 / 2) \sum_{c} \sum_{a} m_{c} m_{a}\left(\mu_{c c a} / z_{c}-\mu_{c a a} /\left|z_{a}\right|\right)\right\} .
\end{aligned}
$$

The parameters appearing in the Equations (19)-(20) are defined as follows:

$$
\begin{gathered}
F=f^{\gamma}+\Sigma \Sigma m_{c} m_{a} B_{c a}^{\prime}+\Sigma \Sigma m_{c} m_{c^{\prime}} \Phi_{c c^{\prime}}^{\prime}+\Sigma \Sigma m_{a} m_{a^{\prime}} \Phi_{a a^{\prime}}^{\prime} \\
f^{\gamma}=-A^{\phi} \cdot\left[I^{1 / 2} /\left(1+1.2 \cdot I^{1 / 2}\right)+\frac{2}{1.2} \cdot \ln \left(1+1.2 \cdot I^{1 / 2}\right)\right] \\
B_{M X}=\beta_{M X}^{0}+\beta_{M X}^{1} \cdot h\left(\alpha_{1} I^{1 / 2}\right)+\beta_{M X}^{2} \cdot h\left(\alpha_{2} I^{1 / 2}\right) \\
h=2[1-(1+x) \exp (-x)] / x^{2} \\
Z=\Sigma m_{i}\left|z_{i}\right|
\end{gathered}
$$

Here " $a$ ", " $c$ " and " $n$ " are cations, anions and neutral species, respectively. $A^{\phi}$ is the Debye-Hueckel limiting slope with numerical values calculated according to an empirical equation suggested by Clegg and Whitfield [16]. For non-associated electrolytes $\alpha_{1}$ and $\alpha_{2}$ take values of 2 and 0, respectively, whereas for 2 - 2 electrolytes the optimized values of $\alpha_{1}$ and $\alpha_{2}$ are 1.4 and $12 \mathrm{~kg}^{1 / 2} \cdot \mathrm{mol}^{-1 / 2}$, respectively. $B^{\prime}$ and $\Phi^{\prime}$ are the ionic strength derivatives of $B$ and $\Phi, I$ is ionic strength, and $\beta_{M X}^{0}, \beta_{M X}^{1}, \beta_{M X}^{2}, C_{M X}, \Phi_{i j}, \psi_{i j k}$, are measurable empirical constants (Pitzer parameters); $\lambda_{i, j}, \mu_{i, j, k}$ are immeasurable second and third viral coefficients, respectively in the Pitzer method. The Equations (19), (20) contain measurable and immeasurable segment. Fortunately, the immeasurable virial coefficients contained in brackets for many cases are not significant and can be neglected. The Pitzer parameters applied in our calculations of the Equations (19), (20) were taken from the literatures which cited elsewhere [17]. Numerical calculations were carried out for 30 - 40 salinity range and for 273 - $298 \mathrm{~K}$ temperature range. Results of calculations were approximated by following empirical relationship. 


$$
\phi_{d} / V \cdot \mathrm{psu}^{-1}=-2.1903 \times 10^{-5}-4.9745 \times 10^{-4} / S+\left(1.3 \times 10^{-7}+2.3578 / S\right) \cdot T / K
$$

\subsection{Calculation of the Barodiffusion Potential in Seawater}

For calculations of Equation (16), needed the partial molal volumes of the major ions of seawater are given as function salinity and temperature elsewhere [18]. Used molar masses of ions are tabulated in the Table 1. Densities of seawater were calculated using EOS of seawater [19]. Numerical calculations of the Equation (16) were carried out for 30 - 40 salinity range and for 273 - $298 \mathrm{~K}$ temperature range. Results of the calculations were approximated by following empirical relationship

$$
\begin{aligned}
\phi_{b d} / V \cdot M^{-1}= & -9.784 \times 10^{-7}-3.9 \times 10^{-9} \cdot S+\left(7.943 \times 10^{-9}+2.24 \times 10^{-11} \cdot S\right) \cdot T / K \\
& -\left(1.795 \times 10^{-11}+6.79 \times 10^{-14} \cdot S\right) \cdot T^{2} / K^{2} .
\end{aligned}
$$

\subsection{Calculation of the Thermodiffusion Potential in Seawater}

Non-isothermal properties of the electrolyte solutions are weakly studied as experimentally and theoretically as well. On this reason, the thermodiffusion properties of the $0.7 \mathrm{~m} \mathrm{NaCl}$ have been used for estimation of the thermodiffusion potential in seawater. In this case Equation (17) is significantly simplified

$$
\varphi_{t d}=\frac{\tau_{\mathrm{Cl}} \cdot \eta_{\mathrm{Cl}}^{*}-\tau_{\mathrm{Na}} \cdot \eta_{\mathrm{Na}}^{*}}{F}
$$

The "absolute" entropies of transfer of sodium and chloride ions for $298 \mathrm{~K}$ have been published elsewhere [20]. The transference numbers in Hittorff's reference frame for 0.7 molality sodium chloride solution have been taken from [21]. Numerical calculation of the Equation (28) gives relationship

$$
\phi_{t d} / V \cdot \operatorname{degr}^{-1}=-4.577 \times 10^{-5}
$$

\section{Discussion}

The scalar fields of the salinity, temperature and gravity cause diffusion of ions. Due to differences in physicochemical properties for each species of the electrolyte solution (mobility, activity coefficients, entropy of transfer, molar masses, and partial molal volume), the diffusion of ions induces electric field inside in seawater. Main feature of the diffusion processes in the electrolyte solutions is fulfillment of electroneutrality on the macroscopic space scale [22]. Another feature is that for diffusion process the electric relaxation time is about $10^{-8} \mathrm{sec}$ or less [23]. Last feature means that during $10^{-8}$ sec electric field becomes steady state after sharp formed scalar fields. On these reasons diffusion-induced electric field can be considered as distribution of dipoles.

With the purpose of an estimation of possible impact $t, S$ fields of the ocean on electric field, the diffusion-induced electric field have been calculated for given $t, S$ profiles ( $t$ is temperature in centigrade scale). These profiles were measured on R/V Sonne S0178 in July 2004 on the slope of the Sakhalin Island (Sea of Okhotsk) (Figure 1(a)). For these profiles, the differences in diffusion potentials between surface and given depth have been calculated using Equations (26), (27), and (29) and results are presented on the Figure 1(b). As it is seen from Figure 1, the order of magnitude of the electric field induced by diffusion has similar order

Table 1. Parameters of seawater used for calculations of the diffusion potential.

\begin{tabular}{cccc}
\hline Ions & $m_{i}(S=35)$ & $\tau_{i}$ & $M_{i}$ \\
\hline $\mathrm{Na}^{+}$ & 0.48616 & 0.2983 & 22.9898 \\
$\mathrm{~K}^{+}$ & 0.01058 & 0.0102 & 39.0980 \\
$\mathrm{Mg}^{2+}$ & 0.05475 & 0.0510 & 24.3050 \\
$\mathrm{Ca}^{2+}$ & 0.01074 & 0.0111 & 40.0780 \\
$\mathrm{Cl}^{-}$ & 0.56918 & 0.5942 & 35.4530 \\
$\mathrm{SO}_{4}^{2-}$ & 0.02927 & 0.0352 & 96.0642 \\
\hline
\end{tabular}



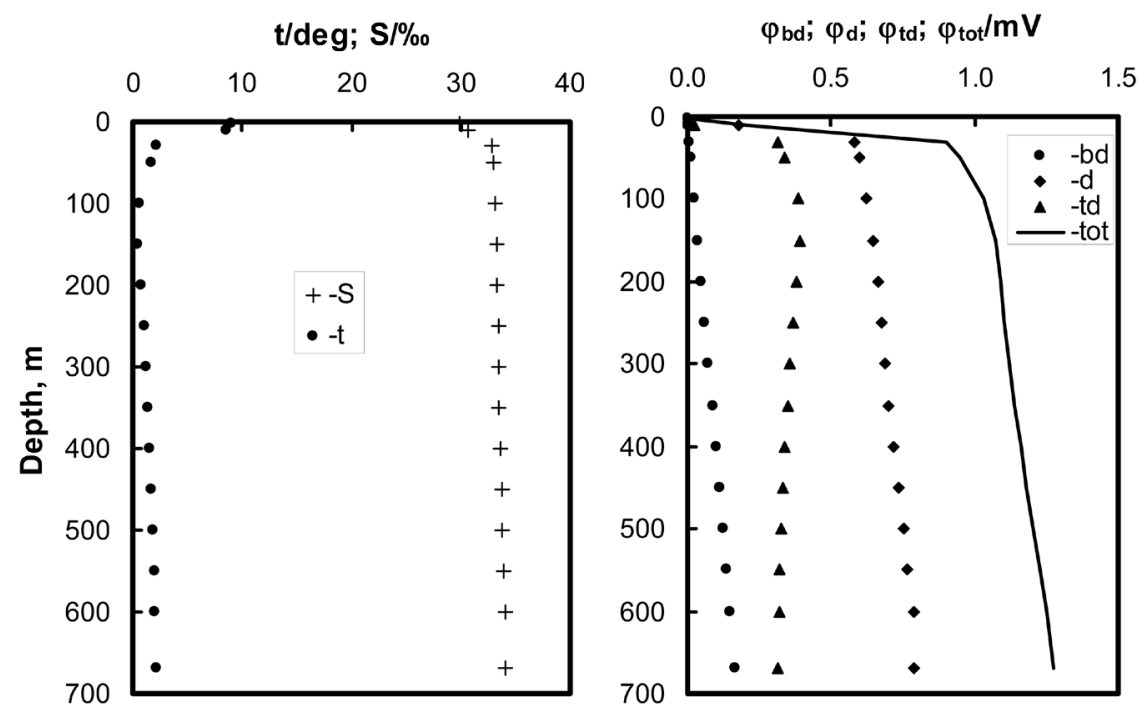

Figure 1. (a) $t, S$ profiles obtained from CTD data on R/V Sonne S0178 in July 2004 (St. 39) on the slope of the Sakhalin Island (Sea of Okhotsk); (b) Calculated profiles of differences in diffusion potentials between surface and given depth using Equations (26), (27), (29).

with those induced by geostrophic currents. Obviously, time-space variations in temperature-salinity structure of the sea have to generate variations in the structure of the electric field. For estimations of these fluctuations, $t$, $S$ profiles obtained for two hydrological stations implemented in 13-th and 15-th August 2004 at the same place $\left(54^{\circ} 26.8^{\prime} \mathrm{N}, 144^{\circ} 4.1^{\prime} \mathrm{E}\right)$ were taken. Variations in $t, S$ profiles were generated by simple subtraction of t, $S$ data between stations 53 (15-th Aug.) and 39 (13-th Aug.), respectively. Figure 2(a) demonstrates them. Since these stations situated in the area of the intermediate water formation of the Okhotsk Sea, then strong time-variations of $t, S$ parameters are observed. Variations of the electrical field corresponded variations of $t, S$ parameters are shown on Figure 2(b).

From Figure 1, Figure 2 it is following that for interpretation of the oceanic electric field measurements, the contribution of diffusion processes into electric field should be taken into account. For this reason, implementation of the electric field measurements should be supplemented by hydrological observations.

Geological and geochemical processes may cause thermal and concentration anomalies, which give anomalies in the electric field. For example, magnetic and electric field variations associated with eruption of volcano where observed and summarized elsewhere [24]. In coastal area incursion of seawater into fresh-water aquifers may to create strong concentration gradients, which induce anomaly of electric field [25]. I think that commonly known in thermodynamic electrolyte solutions, the Equation (13), must be involved into interpretation of observed anomalies of the electric field.

Numerical calculations of Equation (13) in application to seawater have two different sources of uncertainty. One of them is fundamental problem, which may be formulated as impossibility of rigorous determination of thermodynamic properties of the individual ions. It means that thermodynamic properties of electroneutral combination of ions can be determined (measured) only. For example, entropies of transfer, activity coefficients (or derivatives of them), and partial molar volumes for salts $\left(\mathrm{NaCl}, \mathrm{Na}_{2} \mathrm{SO}_{4}\right.$, etc.) in multicomponents of electrolyte solution can be determined but not for individual ions. On this reason extrathermodynamic assumptions are necessary for evaluation of the thermodynamic properties of individual ions. Activity coefficients of the individual ions and them derivatives were calculated by means of Equations (19)-(25) neglecting by last bracket in Equations (19), (20). There are many evidences that adequately experimental uncertainty, the Equations (19) and (20) describe non-ideal behavior of salts for any components of electrolyte solutions (activity coefficients, osmotic coefficients and them derivatives from concentrations, temperature, and pressure). Moreover, contributions of the Pitzer parameters, taking into account interactions between like-charged ions and interactions between three species are very small as rule. It is should be noted that Pitzer parameters are electroneutral combination of the corresponding virial coefficients. The last bracket of the Equations (19), (20) contains just the second virial 


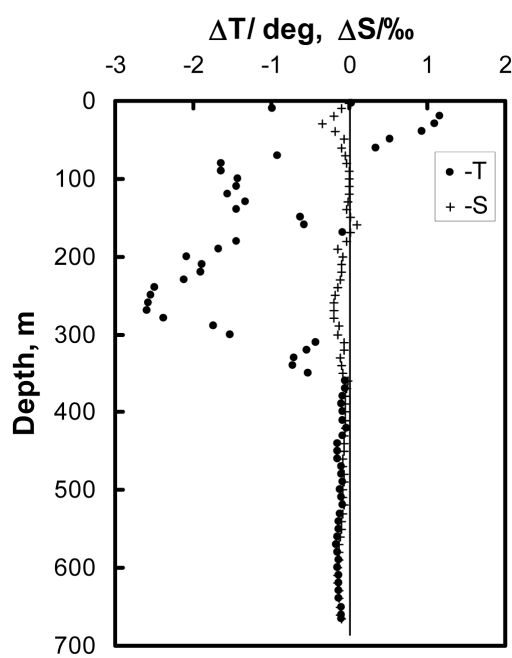

(a)

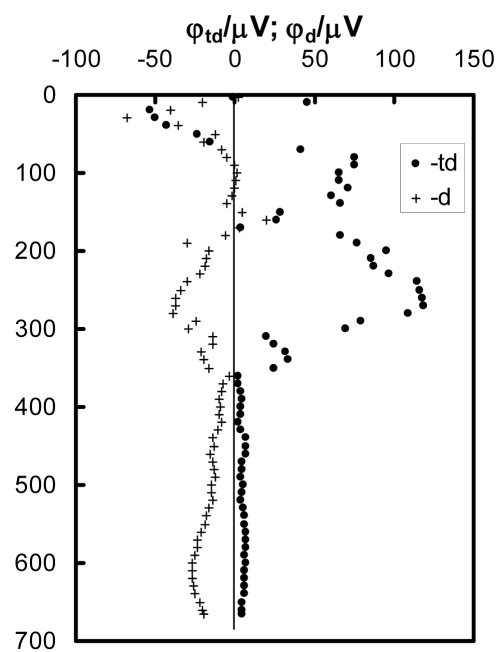

(b)

Figure 2. (a) Variations of $t, S$-profile obtained by simple subtraction of $t$. $S$ data between stations 53 and 39 implemented on R/V Sonne S0178 in 15-th and 13th July, respectively; (b) Variations of the electrical field corresponded variations of $t, S$ parameters.

coefficients taking into account interactions between the same ions (for example, $\mathrm{Na}^{+}-\mathrm{Na}^{+}$or $\mathrm{Cl}^{-}-\mathrm{Cl}^{-}$) and third virial coefficients taking into account triplet interactions. All available experimental data demonstrate that contribution of this type of interactions as in last bracket may be neglected for simple ions such as major ions of seawater and ionic strength less 1 ( $I=0.72$ at $S=35$ ). Another words, the Pitzer method is enough accurate for estimation the activity coefficients and them derivatives for individual ions of seawater. Poisson and Chanu [26] discussed splitting of the partial molar volumes of major seawater salts on ionic constituents. They made a number of assumptions which discussion beyond of this report. De Bethune and Daley [20] obtained ionic entropies of transfer using Agar's reasonable assumption that $\eta_{\mathrm{Cl}^{-}}^{*}=0$ in $0.01 \mathrm{~m} \mathrm{KCl}$ solution.

Another important source of uncertainty of the suggested Equations (26), (27), and (29) is quality of available experimental data. At present time, there is a very good dataset of the Pitzer parameters [27]. Partial molal volumes of sea salts are well studied also [18]. The partial electrical conductances of major ions in seawater were actually measured at $S=38.38$ and $T=298.15 \mathrm{~K}$. However, they are used calculations of transference numbers for 30\% - 40\%o and 273 - $298 \mathrm{~K}$ salinity and temperature ranges, respectively. Using of these transference numbers for expanded salinity and temperature range gives some error in our calculation which difficult to estimate. Thermodiffusion properties of electrolyte solutions are weakly studied, especially in multicomponent electrolyte solutions as experimentally and theoretically as well. Therefore, estimation gradient of electric potential induced by temperature gradient by means of relationship (29) is rather qualitative. There is way of "experimental determination" of contributions of salinity, temperature, and pressure gradients into formation of the electric potential gradient in seawater. I take "experimental determination" into inverted commas because it was below suggested that experiments should be supplemented additional extrathermodynamic assumptions.

I suggest to measure diffusion, thermodiffusion, and barodiffusion potential by means of measurements of electromotive forces (EMF) following cells:

\begin{tabular}{|c|c|c|c|}
\hline g, ${\mathrm{AgCl}-\mathrm{Cl}^{-}}^{-}$ & Synthetic seawater, $\left(S_{1}\right)$ & || Synthetic seawater, $\left(S_{2}\right)$ & \\
\hline g, AgCl-C & Synthetic seawater, $\left(T_{1}\right)$ & $\left(T_{2}\right)$ & \\
\hline r & Synthetic seawater, $\left(P_{1}\right.$ & | Synthetic seawater, $\left(P_{2}\right)$ & A \\
\hline
\end{tabular}

EMF of the cells (A), (B), (C) may be writing respectively:

$$
E_{\mathrm{A}}=\frac{R T}{F} \ln \left(m_{1}^{\mathrm{Cl}} / m_{2}^{\mathrm{Cl}}\right)+\frac{R T}{F} \ln \left(\gamma_{1}^{\mathrm{Cl}} / \gamma_{2}^{\mathrm{Cl}}\right)_{T, P}+\int_{S_{1}}^{S_{2}} \phi_{d} \mathrm{~d} S
$$




$$
\begin{gathered}
E_{B}=\int_{T_{1}}^{T_{2}} \frac{\partial\left(\phi_{\mathrm{Ag}, \mathrm{AgCl}}^{\mathrm{o}}\right)_{P}}{\partial T} \mathrm{~d} T+\frac{R \mathrm{~d} T}{F} \ln \left(\gamma_{1}^{\mathrm{Cl}} / \gamma_{2}^{\mathrm{Cl}}\right)_{S, P}-\frac{R T}{F} \int_{T_{1}}^{T_{2}} \frac{\partial\left(\ln \gamma^{\mathrm{Cl}}\right)_{S, P}}{\partial T} \mathrm{~d} T+\int_{T_{1}}^{T_{2}} \phi_{\mathrm{td}} \mathrm{d} T \\
E_{C}=\int_{P_{1}}^{P_{2}} \frac{\partial\left(\phi_{\mathrm{Ag}, \mathrm{AgCl}}^{o}\right)_{T}}{\partial P} \mathrm{~d} P-\frac{R T}{F} \int_{P_{1}}^{P_{2}} \frac{\partial\left(\ln \gamma^{\mathrm{Cl}}\right)_{S, T}}{\partial P} \mathrm{~d} P+\int_{P_{1}}^{P_{2}} \phi_{b d} \mathrm{~d} P
\end{gathered}
$$

where, $\partial\left(\phi_{\mathrm{Ag}, \mathrm{AgCl}}^{o}\right)_{P} / \partial T$ and $\partial\left(\phi_{\mathrm{Ag}, \mathrm{AgCl}}^{o}\right)_{T} / \partial P$ are respectively, temperature and pressure coefficients of the following electrode reaction:

$$
\mathrm{AgCl}_{s}+\mathrm{e}^{-} \rightarrow \mathrm{Ag}_{s}+\mathrm{Cl}^{-}(\mathrm{aq})
$$

Here, subscription " $s$ " means solid. I suggest using of rather synthetic seawater than natural seawater because natural seawater contains $\mathrm{Br}^{-}$and $\mathrm{I}^{-}$ions which interference potential of silver-silver chloride electrode. Estimation diffusion, thermodiffusion and barodiffusion potentials via measurements of the EMF of cells (A)-(C) is shortest way with minimum amount of extrathermodynamic assumptions. However, noise of the silver electrode does not permit to carry out accurate measurements of the $E_{C}$ even when seawater column would has a few meters because expected measured magnitude has order about $10^{-6} \mathrm{~V}$. In this case centrifugal cells should be used [28]. It is should be noted that contribution of barodiffusion into electric field of the ocean is almost constant (about $-3 \times 10^{-7} \mathrm{~V} / \mathrm{M}$ ) and in many cases of electrical fields study it can be negligible.

\section{Conclusions}

Electrochemical processes such as diffusion, thermodiffusion, and barodiffusion induce electric field in the ocean due to existence of natural salinity, temperature, and gravitational fields. Diffusion-induced electric field can be considered as distribution of dipoles.

Natural variations of hydrological properties cause variations of electric field in seawater.

Geological and geochemical processes, which induce thermal and concentration anomalies, may result in anomalies in the electric field.

Using of the chemical model of seawater, the Pitzer method for calculations of non-ideal behavior of electrolyte solutions, and available thermodynamic data for major ions of seawater, the numerical calculation of Equation (13) was carried out. Results of numerical calculations are represented by empirical relationships which are easy to apply to any modeling.

Modern theoretical and experimental knowledge of the thermodynamic properties of multicomponent electrolyte solutions does not permit to quantitatively describe diffusion-induced electric field in the ocean. On this reason it is suggested to carry out special potentiometric experiments with synthetic seawater for accurate estimation diffusion-induced electric field in seawater.

\section{References}

[1] Palshin, N.A., Vanyan, L.L. and Kaikkonen, P. (1996) On-Shore Amplification of the Electric Field Induced by a Coastal Sea Current. Physics of the Earth and Planetary Interiors, 94, 269-273. http://dx.doi.org/10.1016/0031-9201(95)03098-0

[2] Palshin, N.A. (1996) Oceanic Electromagnetic Studies: A Review. Surveys in Geophysics, 17, 455-491. http://dx.doi.org/10.1007/BF01901641

[3] Longuet-Higgins, M.S., Stern, M.E. and Stommel, H. (1954) The Electrical Field Induced by Ocean Currents and Waves with Applications to Method of Towed Electrodes. Physical Oceanography and Meteorology, $13,37$.

[4] Cox, C. (1980) Electromagnetic Induction in the Oceans and Inferences of the Constitution of the Earth. Surveys in Geophysics, 4, 137-156. http://dx.doi.org/10.1007/BF01452963

[5] Semenchenko, V.K. (1941) Physical Theory of the Solutions. Leningrad, Moscow. (In Russian)

[6] Tishchenko, P.Ya. (1984) Contribution of Diffusion Processes into Formation of Electric Field of the Ocean. Dokladi Akademii Nauk, 279, 1234-1238. (In Russian)

[7] Tishchenko, P.Ya. (1991) EMF Method for Measurements of the Electric Field in the Ocean. VINITI, Moscow, No. 165-B91. (In Russian) 
[8] De Groot, S.R. and Mazur, P. (1962) Non-Equilibrium Thermodynamics. North-Holland Publishing Co., Amsterdam.

[9] Kirkwood, J.G., Baldwin, R.L., Dunlop, P.J., Gosing, L.J. and Kegeles, G. (1960) Flow Equations and Frames of Reference for Isothermal Diffusion in Liquids. Journal of Chemical Physics, 33, 1505-1513. http://dx.doi.org/10.1063/1.1731433

[10] Agar, J.N. (1963) Thermogalvanic Cells. In: Delahay, P., Ed., Advances in Electrochemistry and Electrochemical Engineering, Interscience, New York, 31-120.

[11] De Groot, S.R. (1951) Thermodynamics of Irreversible Processes. North-Holland Publishing Comp., Amsterdam.

[12] DOE (1994) Handbook of Methods for the Analysis of the Various Parameters of the Carbon Dioxide System in Sea water. Version 2, Dickson, A.G. and Goyet, C., Eds., ORNL/CDIAC-74, Oak Ridge, Tennessee.

[13] Poisson, A., Perie, M., Perie, J. and Chemla, M. (1979) Individual Equivalent Conductances of the Major Ions in Seawater. Journal of Solution Chemistry, 8, 377-394. http://dx.doi.org/10.1007/BF00646790

[14] Pitzer, K.S. (1973) Thermodynamics of Electrolytes. I. Theoretical Basis and General Equations. Journal of Physical Chemistry, 77, 268-277. http://dx.doi.org/10.1021/j100621a026

[15] Pitzer, K.S. (1991) Ionic Interaction Approach: Theory and Data Correlation. In: Pitzer, K.S., Ed., Activity Coefficients in Electrolyte Solutions, CRC Press, Roca Raton, 75-153.

[16] Clegg, S.L. and Whitfield, M. (1991) Activity Coefficients in Natural Waters. In: Pitzer, K.S., Ed., Activity Coefficients in Electrolyte Solutions, CRC Press, Roca Raton, 279-434.

[17] Tishchenko, P., Hensen, C., Wallmann, K. and Wong, C.S. (2005) Calculation of the Stability and Solubility of Methane Hydrate in Seawater. Chemical Geology, 219, 37-52. http://dx.doi.org/10.1016/j.chemgeo.2005.02.008

[18] Poisson, A. and Chanu, J. (1980) Semi-Empirical Equations for the Partial Molar Volumes of Some Ions in Water and in Seawater. Marine Chemistry, 8, 289-298. http://dx.doi.org/10.1016/0304-4203(80)90018-3

[19] Fofonoff, N.P. and Millard, R.C. (1983) Algorithms for Computation of Fundamental Properties of Seawater. UNESCO, Paris.

[20] De Bethune, A.J. and Daley, H.O. (1969) The Thermal Temperature Coefficient of the Calomel Electrode Potential between $0^{\circ} \mathrm{C}$ and $70^{\circ} \mathrm{C}$. II. Thermodynamic Results-The Moving and Transport Entropies and Heat Capacities of Aqueous Chloride Salines and Their Ions. Journal of the Electrochemical Society, 116, 1395-1401. http://dx.doi.org/10.1149/1.2411532

[21] Kajmakov, E.A. and Varshavskaja, N.L. (1966) Measurements of Transference Numbers in Aqueous Solution Electrolytes. Uspekhi Khimii, 35, 201-228. (In Russian)

[22] Aguilella, V.M., Mafe, S. and Pellicer, J. (1987) On the Nature of the Diffusion Potential Derived from Nernst-Planck Flux Equations by Using the Electroneutrality Assumption. Electrochimica Acta, 32, 483-488. http://dx.doi.org/10.1016/0013-4686(87)85018-1

[23] Hafemann, D.R. (1965) Charge Separation in Liquid Junction. Journal of Physical Chemistry, 69, 4226-4231. http://dx.doi.org/10.1021/j100782a027

[24] Sasai, Y., Uyeshima, M., Zlotnicki, J., Utada, H., Kagiyama, T., Hashimoto, T. and Takahashi, Y. (2002) Magnetic and Electric Field Observations during the 2000 Activity of Miyake-Jima Volcano, Central Japan. Earth and Planetary Science Letters, 203, 769-777. http://dx.doi.org/10.1016/S0012-821X(02)00857-9

[25] Nobes, D.C. (1996) Troubled Waters: Environmental Applications of Electrical and Electromagnetic Methods. Surveys in Geophysics, 17, 393-454. http://dx.doi.org/10.1007/BF01901640

[26] Poisson, A. and Chanu, J. (1976) Partial Molal Volumes of Some Major Ions in Seawater. Limnology and Oceanography, 21, 853-861. http://dx.doi.org/10.4319/lo.1976.21.6.0853

[27] Millero, F.J. and Pierrot, D. (1988) A Chemical Equilibrium Model for Natural Waters. Aquatic Geochemistry, 4, 153-199. http://dx.doi.org/10.1023/A:1009656023546

[28] Miller, D.G. (1956) Thermodynamic Theory of Irreversible Processes. II. Sedimentation Equilibrium of Fluids in Gravitational and Centrifugal Fields. American Journal of Physics, 24, 535-561. 